\title{
Prediction of peak flow values followed by feedback improves perception of lung function and adherence to inhaled corticosteroids in children with asthma
}

\author{
Jonathan M Feldman, ${ }^{1,2}$ Haley Kutner, ${ }^{1}$ Lynne Matte, ${ }^{1}$ Michelle Lupkin, ${ }^{1}$ \\ Dara Steinberg, ${ }^{1}$ Kimberly Sidora-Arcoleo, ${ }^{3}$ Denise Serebrisky, ${ }^{4}$ Karen Warman ${ }^{5}$
}

\begin{abstract}
${ }^{1}$ Ferkauf Graduate School of Psychology, Yeshiva University, Bronx, New York, USA 2Department of Epidemiology and Population Health, Albert Einstein College of Medicine, Bronx, New York, USA

${ }^{3}$ College of Nursing, Ohio State University, Columbus, Ohio, USA

${ }^{4}$ Department of Pediatrics, Jacobi Medical Center, Bronx, New York, USA

${ }^{5}$ Department of Pediatrics, Children's Hospital at

Montefiore, Albert Einstein College of Medicine, Bronx, New York, USA
\end{abstract}

Correspondence to Jonathan M. Feldman, Ferkauf Graduate School of Psychology/ Yeshiva University, Rousso Building, 1300 Morris Park Avenue, Bronx, NY 10461 USA; jonathan.feldman@ einstein.yu.edu

Received 18 February 2012 Accepted 9 October 2012

\begin{abstract}
Background Failure to detect respiratory compromise can lead to emergency healthcare use and fatal asthma attacks. The purpose of this study was to examine the effect of predicting peak expiratory flow (PEF) and receiving feedback on perception of pulmonary function and adherence to inhaled corticosteroids (ICS).
\end{abstract}

Methods The sample consisted of 192 ethnic minority, inner-city children (100 Puerto Rican, 54 AfricanAmerican, 38 Afro-Caribbean) with asthma and their primary caregivers recruited from outpatient clinics in Bronx, New York. Children's PEF predictions were entered into an electronic spirometer and compared with actual PEF across 6 weeks. Children in one study were blinded to PEF ( $n=88$; no feedback) and children in a separate study were able to see PEF ( $n=104$; feedback) after predictions were locked in. Dosers were attached to asthma medications to monitor use.

Results Children in the feedback condition displayed greater accuracy $(p<0.001)$, less under-perception $(p<0.001)$ and greater over-perception $(p<0.001)$ of respiratory compromise than children in the no feedback condition. This between-group difference was evident soon after baseline training and maintained across 6 weeks. The feedback condition displayed greater adherence to ICS $(p<0.01)$ and greater quick-relief medication use $(p<0.01)$ than the no feedback condition.

Conclusions Feedback on PEF predictions for ethnic minority, inner-city children may decrease underperception of respiratory compromise and increase adherence to controller medications. Children and their families may shift their attention to asthma perception and management as a result of this intervention.

\section{INTRODUCTION}

Under-perception of respiratory compromise is a risk factor for emergency department (ED) visits, hospitalisations, near-fatal and fatal asthma attacks in children. ${ }^{1}$ Children with severe bronchial hyperresponsiveness are particularly at risk for missing the early stage of bronchoconstriction during asthma exacerbations. ${ }^{2}$ A window of opportunity exists when patients with asthma can use bronchodilator medications in the days leading up to a severe asthma exacerbation and prevent its onset. $^{3}{ }^{4}$ However, there has been very limited research on how to improve the perceptual accuracy of pulmonary function.

Peak expiratory flow (PEF) meters are recommended for asthma self-management in individuals

\section{Key messages}

What is the key question?

- Do children with asthma who receive feedback on predictions of peak expiratory flow (PEF) have better perception of pulmonary function and greater adherence to inhaled corticosteroids (ICS) than children who do not receive feedback?

What is the bottom line?

- Children who received feedback on predictions of PEF were less likely to under-perceive respiratory compromise and were more adherent to ICS than children who did not receive feedback.

\section{Why read on?}

- Prediction of PEF values followed by feedback may assist children with asthma in improving the accuracy of disease perception and ICS adherence.

with inaccurate asthma symptom perception. ${ }^{5} \mathrm{PEF}$ monitoring typically involves measurement and recording of PEF twice per day prior to taking asthma medications. PEF prediction with feedback requires children to guess their PEF, which is locked in, and then see their actual PEF. This protocol can be more interactive and engaging for children than simply monitoring PEF, as they strive to see how close their guesses match their actual PEF. A small, uncontrolled study of PEF prediction-feedback showed that children and adults demonstrated accurate PEF predictions soon after baseline training, which was maintained across 3 weeks. ${ }^{6}$ Receiving feedback on PEF using electronic devices is linked with better adherence to inhaled corticosteroid (ICS) medications and less asthma morbidity in adults. ${ }^{7}$ Immediate, objective feedback following PEF prediction may alter an individual's attention and increase the perceived threat level, which may play a key role in asthma symptom perception. ${ }^{8}$ Motivational interviewing is based upon self-perception theory ${ }^{9}$ and posits that objective feedback showing low PEF values can be used to elicit motivational statements from the client related to the benefits of long-term asthma control and adherence to ICS medications. These verbalisations by the patient in turn predict attitudinal and behavioural changes toward these goals. ${ }^{10}$ 
The Bronx, New York is one of the poorest counties in the USA, and paediatric asthma prevalence and hospitalisation rates are among the highest in the country. ${ }^{11}$ Puerto Rican and non-Latino black children have the highest paediatric asthma prevalence rates compared with other racial/ethnic groups. ${ }^{12}$ Perception of pulmonary function ${ }^{13}$ and adherence to ICS medications ${ }^{15} 16$ is worse in Puerto Rican and African-American children versus Caucasian children. Underestimation of asthma severity by providers and under-prescription of ICS medications contribute to poor adherence rates among inner-city children. ${ }^{17}$ We hypothesised that children who receive feedback on PEF predictions would have less under-perception of respiratory compromise, better ICS adherence and less quick-relief medication use than children who do not receive feedback.

\section{METHODS}

\section{Participants}

A convenience sample of children with asthma and their primary caregivers in the Bronx, New York was recruited from asthma and primary care clinics, the ED and mailings. Children in the first study received PEF feedback following PEF prediction and ranged in age from 7 to 15 years old. Children in the second study did not receive PEF feedback following PEF prediction and ranged in age from 7 to 12 years old. All other inclusion criteria were identical between the two studies. A physician diagnosis of asthma was confirmed by medical chart review and children were required to report breathing difficulties within the past year. Only Puerto Rican, African-American and Afro-Caribbean children were included and one child per family was eligible to participate. Children were excluded for cognitive learning disability, vocal cord dysfunction (assessed by chart review and spirometry) or inability to conduct spirometry. Families were financially compensated for completing the training session and measures at baseline, and returning devices 6 weeks later. The institutional review board at Albert Einstein College of Medicine approved the study protocol and consent process.

\section{Measures}

Pulmonary function perception

Children and their primary caregivers were trained extensively during a baseline session on use of the AM2 electronic spirometer and diary (ERT, Philadelphia, Pennsylvania, USA). A colourful sticker was placed on the AM2 with the child's predicted PEF values corresponding to the three traffic light zones ranging from a happy face to a sad face. Children entered their prediction of PEF into the AM2 and then conducted a forced vital capacity manoeuvre. Although parents were encouraged to enforce the child's use of the AM2, the actual prediction of PEF was from the child. All children practiced maximal effort blows until they consistently displayed correct technique. Each use of the AM2 required three consecutive manoeuvres and the best of the three blows was retained. Participants were instructed to use the AM2 device before taking any asthma medications each morning and evening for 6 weeks at home. An acoustic alarm served as a reminder to use the device at consistent times each day. Children in the PEF feedback condition were able to see their PEF values. However, their predictions of PEF were locked in before conducting the blow and could not be changed. Children in the no feedback condition were blinded to PEF during the 6 weeks and only saw their PEF during the baseline session.

The Asthma Risk Grid ${ }^{18}$ (figure 1) was developed to measure perception of lung function. Children's PEF prediction and actual PEF (both in litres/min) are converted to percent of objective personal best for each individual child. The personal best PEF for each child is based on the highest PEF achieved during 6 weeks of data collection after outliers are removed. The percentage of predictions in the accurate zone (close match between subjective and objective), danger zone (underperception of respiratory compromise) and magnification zone (over-perception of respiratory compromise) are the measures of perception. For example, a child's prediction of PEF that corresponds to $85 \%$ personal best, but actual PEF value was $55 \%$ personal best would be categorised in the danger zone. The smallest margin of error allowed is $10 \%$, as depicted in the $\pm 10 \%$ wedge of the accurate zone in figure 1 . Each blow is categorised into only one of these three zones. A child is not categorised as an 'under perceiver' or 'over perceiver'. For example, a child might be categorised $50 \%$ of the time in the accurate zone and $25 \%$ in the danger and magnification zones. Percentages are treated as continuous variables in each zone for each child.

This methodology for perception of pulmonary function has been used in several studies, although children were not blinded to $\mathrm{PEF}^{13}{ }^{19}$ Greater perceptual accuracy of pulmonary function
Figure 1 Asthma risk grid (adapted with permission from OceanSide Publications, Inc from Klein et $a l^{18}$ ). Accurate zone: boxes 1, 5, 9 and $\pm 10 \%$ wedge; magnification zone: boxes 2, 3 and 6; danger zone: boxes 4, 7 and 8. PEF, peak expiratory flow.

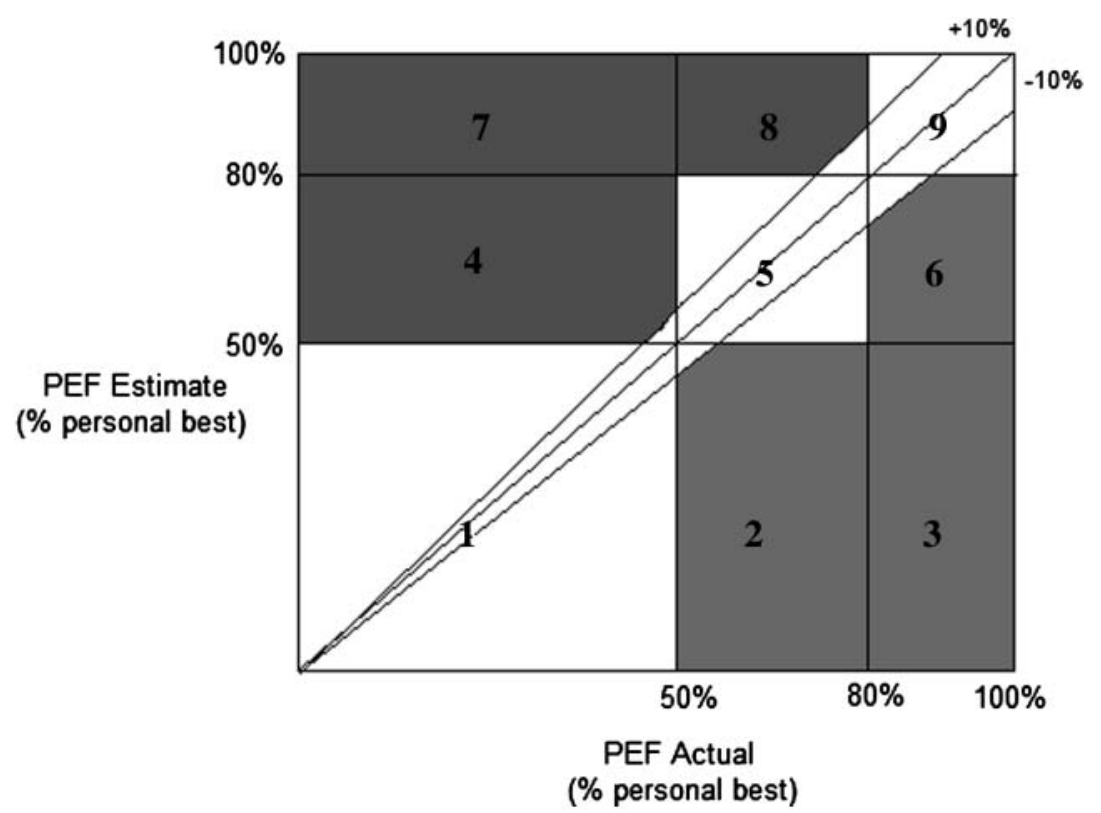


using this methodology is associated with lower levels of asthma morbidity during the past year ${ }^{13}{ }^{19}$ and prospectively across a 1-year follow-up. ${ }^{20}$

\section{Adherence to ICS and quick-relief medication use during the perception task}

Doser devices (MediTrack Products, Hudson, Massachusetts, USA) were attached to the top of ICS and quick-relief metered dose inhalers. Dosers are more reliable for monitoring medication use than self-report or pharmacy records. ${ }^{21}$ These devices record the number of actuations taken per day across the past 30 days. Adherence to ICS medications was calculated by dividing the number of puffs taken by the number of puffs prescribed. Data reduction steps eliminated any extra puffs recorded to avoid artificial inflation of adherence to ICS or quick-relief medication use. Dosers were assigned for ICS medications to 95 of 192 children, and for quick-relief medication to 181 of 192 children. Dosers for ICS and quick-relief medications were lost by 10 and 16 children, respectively.

\section{Asthma severity, asthma control and asthma morbidity at baseline}

Asthma severity ratings were based on national guidelines and incorporated asthma symptoms/impairment (eg, nocturnal awakenings, quick-relief medication use), current asthma medication regimen and pulmonary function (\% forced expiratory volume in $\left.1 \mathrm{~s}\left(\mathrm{FEV}_{1}\right)\right)$ at baseline. ${ }^{5}$ Asthma control ratings were based on symptoms/impairment and pulmonary function. Severity and control were based on the most severe level reached in each individual category. Spirometry was conducted using a clinical spirometer (nSpire Health, Longmont, Colorado, USA) according to American Thoracic Society standards. ${ }^{22}$ The Asthma Functional Severity Scale ${ }^{23}$ assessed caregiver report of asthma symptoms/impairment during the month prior to the study. The scale has good reliability and validity $^{23}$ and was translated and adapted into Spanish for Latinos. ${ }^{13}$ Caregiver reports of ED visits and hospitalisations for asthma were based on the past year.

\section{Language preference and poverty threshold}

The language of the interview was conducted in English or Spanish based on the caregiver's preference. Families were categorised as living above or below the federal poverty line. ${ }^{24}$

\section{Data analysis}

Analyses of covariance were conducted to assess differences between the PEF feedback and no feedback conditions on each of the perception zones and medication use. The child's age was entered as a covariate due to the difference in age range between the two conditions, and older children are more accurate on perception of pulmonary function ${ }^{13}$ but less adherent to controller medications. ${ }^{25}$ Main analyses were repeated by restricting the age range to $7-12$ years old for the entire sample to ensure that any between-group differences were not explained by age. A growth model with individually varying times of observation examining accuracy over time was conducted using MPlus V6.1. This analysis examined whether a learning effect across the 6-week period was associated with the PEF feedback versus no feedback condition. Ethnicity and child's age were included as covariates. The perceptual accuracy protocol requires a minimum of 20 data points to provide reliable estimates of perceptual ability. ${ }^{13}$ Tukey-Kramer adjustment was specified when multiple comparison tests were conducted. Analyses were conducted with PASW Statistics V.18 and SAS V.9.2.

\section{RESULTS \\ Participants}

A total of 192 children completed the study (see figure 2 ). The rate of completion $(88.1 \%$ for PEF feedback vs $78.6 \%$ for no feedback) was not significantly different between the two conditions. Children who completed the study were not different according to ethnicity, age or sex versus children who did not complete the study, across both conditions (PEF feedback, no feedback). The primary reasons for ineligibility included ethnicity and learning disability in the child. Participants were recruited via asthma clinics (51.8\%), mailings (22.6\%), primary care clinics $(12.8 \%)$, ED $(6.7 \%)$ and other sources $(6.1 \%)$. Table 1 shows that children in the PEF feedback condition were older than the no feedback condition, which was the result of the wider age range in the feedback condition. No other between-group differences were found on demographics, recruitment site, asthma control or asthma severity. Asthma severity was mostly in the moderate and severe persistent categories, which is consistent with asthma clinics being the primary source of recruitment.
Figure 2 Participant enrolment in the peak expiratory flow (PEF) feedback and no feedback conditions.

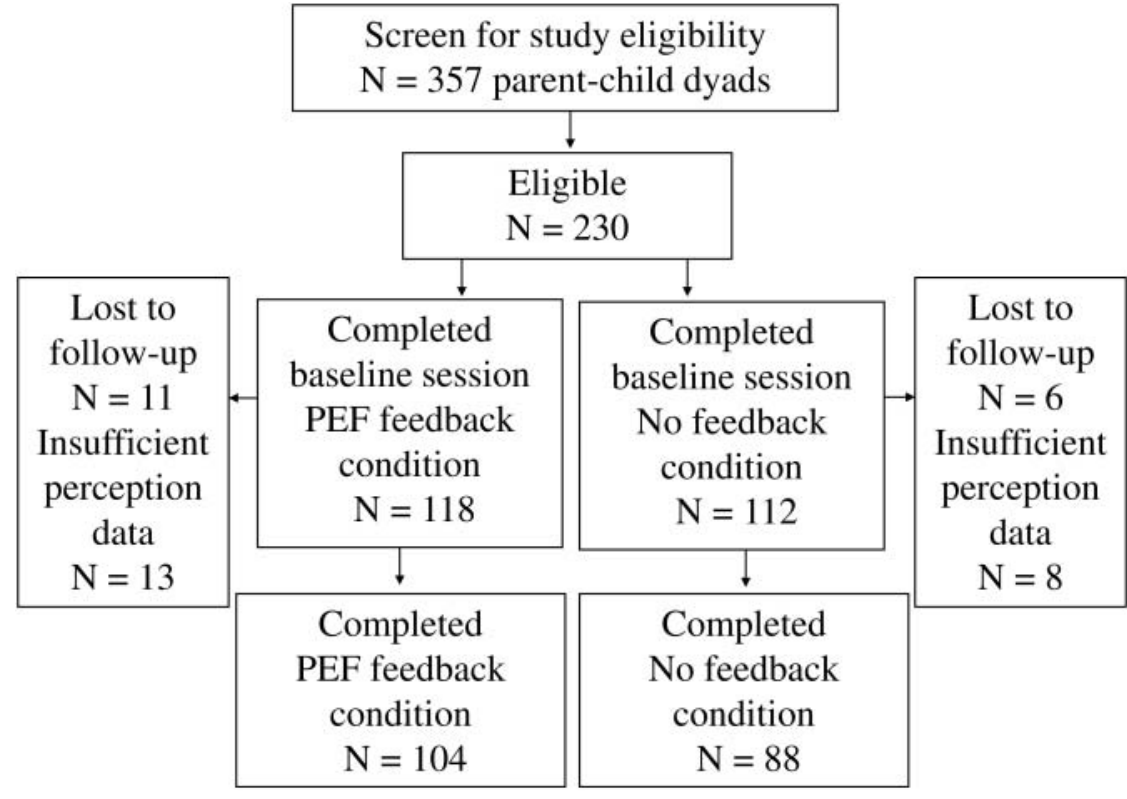


Table 1 Baseline characteristics of participants by PEF feedback condition

\begin{tabular}{|c|c|c|c|}
\hline & $\begin{array}{l}\text { PEF feedback } \\
(n=104)\end{array}$ & $\begin{array}{l}\text { No PEF feedback } \\
(n=88)\end{array}$ & p Value \\
\hline Age of child, mean $\pm S E$ & $10.8 \pm 0.2$ & $9.4 \pm 0.2$ & $<0.001$ \\
\hline Sex of child, $\%$ male & 55.8 & 59.1 & 0.643 \\
\hline \multicolumn{4}{|l|}{ Ethnicity, \% } \\
\hline Puerto Rican $(n=100)$ & 50.0 & 54.5 & \multirow[t]{3}{*}{0.801} \\
\hline African-American $(n=54)$ & 28.8 & 27.3 & \\
\hline Afro-Caribbean $(\mathrm{n}=38)$ & 21.2 & 18.2 & \\
\hline \multicolumn{4}{|l|}{ Primary caregiver } \\
\hline Biological mother, \% & 87.5 & 88.6 & 0.809 \\
\hline Below poverty threshold, \% & 54.5 & 43.2 & 0.132 \\
\hline \multicolumn{4}{|l|}{ Maternal education } \\
\hline At least some college & 40.3 & 40.9 & \multirow[t]{3}{*}{0.609} \\
\hline High school & 24.8 & 21.6 & \\
\hline Less than high school & 34.9 & 37.5 & \\
\hline English parent interview, \% & 89.4 & 95.5 & 0.121 \\
\hline US-born parent, $\%$ & 63.5 & 63.5 & 0.992 \\
\hline \multicolumn{4}{|l|}{ Recruitment site, $\%$} \\
\hline Asthma clinic & 54.2 & 48.9 & \multirow[t]{2}{*}{0.450} \\
\hline Other recruitment site & 45.8 & 51.1 & \\
\hline Taking ICS medication, \% & 62.0 & 57.6 & 0.373 \\
\hline Oral steroid burst (past year), \% & 61.5 & 60.2 & 0.852 \\
\hline \multicolumn{4}{|l|}{ Asthma severity, \% } \\
\hline Intermittent/mild persistent & 12.9 & 10.6 & \multirow[t]{3}{*}{0.680} \\
\hline Moderate persistent & 55.9 & 62.4 & \\
\hline Severe persistent & 31.2 & 27.1 & \\
\hline \multicolumn{4}{|l|}{ Asthma control, \% } \\
\hline Well controlled & 10.8 & 5.9 & \multirow[t]{3}{*}{0.475} \\
\hline Not well controlled & 54.8 & 55.3 & \\
\hline Very poorly controlled & 34.4 & 38.8 & \\
\hline
\end{tabular}

ICS, inhaled corticosteroids; PEF, peak expiratory flow.
Table 2 Perception of pulmonary function, demographics and asthma variables (controlling for age)

\begin{tabular}{|c|c|c|c|}
\hline & $\begin{array}{l}\text { Accurate } \\
\text { zone }(\%)\end{array}$ & $\begin{array}{l}\text { Danger } \\
\text { zone }(\%)\end{array}$ & $\begin{array}{l}\text { Magnification } \\
\text { zone }(\%)\end{array}$ \\
\hline \multicolumn{4}{|l|}{ Sex of child } \\
\hline Male $(n=110)$ & $55.0 \pm 2.2$ & $26.6 \pm 2.3$ & $18.4 \pm 2.1$ \\
\hline Female $(n=82)$ & $55.0 \pm 2.6$ & $28.3 \pm 2.7$ & $16.8 \pm 2.4$ \\
\hline p Value & 0.991 & 0.638 & 0.609 \\
\hline \multicolumn{4}{|l|}{ Ethnicity } \\
\hline Puerto Rican $(\mathrm{n}=100)$ & $56.3 \pm 2.3$ & $28.4 \pm 2.4$ & $15.2 \pm 2.2$ \\
\hline African-American $(n=54)$ & $57.6 \pm 3.2$ & $23.6 \pm 3.3$ & $18.8 \pm 3.0$ \\
\hline Afro-Caribbean $(n=38)$ & $47.7 \pm 3.8$ & $29.6 \pm 4.0$ & $22.6 \pm 3.6$ \\
\hline $\mathrm{p}$ Value & 0.101 & 0.409 & 0.189 \\
\hline \multicolumn{4}{|l|}{ Poverty threshold } \\
\hline Below $(n=90)$ & $55.4 \pm 2.5$ & $26.5 \pm 2.6$ & $18.1 \pm 2.4$ \\
\hline Above $(n=92)$ & $54.5 \pm 2.4$ & $27.1 \pm 2.5$ & $18.3 \pm 2.3$ \\
\hline $\mathrm{p}$ Value & 0.806 & 0.868 & 0.939 \\
\hline \multicolumn{4}{|l|}{ Parent nativity } \\
\hline US-born parent $(n=120)$ & $57.6 \pm 2.1$ & $26.4 \pm 2.2$ & $16.0 \pm 2.0$ \\
\hline Foreign-born parent $(n=69)$ & $50.2 \pm 2.8$ & $28.4 \pm 2.9$ & $21.4 \pm 2.6$ \\
\hline $\mathrm{p}$ Value & 0.035 & 0.592 & 0.103 \\
\hline \multicolumn{4}{|l|}{ Asthma severity } \\
\hline $\begin{array}{l}\text { Intermittent/mild persistent } \\
(\mathrm{n}=21)\end{array}$ & $50.3 \pm 5.1$ & $24.6 \pm 5.4$ & $25.0 \pm 4.8^{*}$ \\
\hline Moderate persistent $(n=105)$ & $53.8 \pm 2.3$ & $26.5 \pm 2.4$ & $19.7 \pm 2.1$ \\
\hline Severe persistent $(n=52)$ & $56.6 \pm 3.2$ & $32.2 \pm 3.4$ & $11.1 \pm 3.0^{*}$ \\
\hline $\mathrm{p}$ Value & 0.556 & 0.315 & 0.020 \\
\hline \multicolumn{4}{|l|}{ ICS medication use } \\
\hline Yes $(n=115)$ & $56.6 \pm 2.2$ & $26.2 \pm 2.3$ & $17.2 \pm 2.1$ \\
\hline No $(n=77)$ & $52.6 \pm 2.7$ & $28.9 \pm 2.8$ & $18.5 \pm 2.5$ \\
\hline $\mathrm{p}$ Value & 0.249 & 0.454 & 0.693 \\
\hline
\end{tabular}

Values represent mean $\pm S E$ of the mean.

*Intermittent/mild persistent versus severe persistent, $p=0.04$

ICS, inhaled corticosteroids.

create the same age range in both conditions. Children who received feedback $(n=75)$ were still less likely to under-perceive $(\mathrm{F}(1,160)=58.29, \mathrm{p}<0.001)$ and more likely to over-perceive $(\mathrm{F}(1,160)=18.91, \mathrm{p}<0.001)$ respiratory compromise, and had higher accurate zone scores $(F(1,160)=10.29, p=0.002)$ than children without feedback $(n=88)$.

The results of the growth model showed that there was no evidence of an incremental learning effect across time in either the feedback or no feedback condition. The data demonstrated good fit with the model: root mean square error approximation $($ RMSEA $)=0.03$, comparative fit index $(\mathrm{CFI})=0.94$ and TuckerLewis index $(\mathrm{TLI})=0.94$. The results revealed that there were

Table 3 Feedback condition, perception of pulmonary function, and asthma medication use across the perception trial (adjusted for child's age)

\begin{tabular}{lcrr}
\hline & $\begin{array}{l}\text { PEF } \\
\text { feedback }\end{array}$ & \multicolumn{1}{c}{$\begin{array}{l}\text { No PEF } \\
\text { feedback }\end{array}$} & p Value \\
\hline Accurate zone, \% & $60.7 \pm 2.3$ & $48.2 \pm 2.5$ & $<0.001$ \\
Danger zone, \% & $15.3 \pm 2.1$ & $41.6 \pm 2.3$ & $<0.001$ \\
Magnification zone, \% & $24.0 \pm 2.1$ & $10.2 \pm 2.3$ & $<0.001$ \\
Prediction of PEF, \% personal best & $73.0 \pm 0.4$ & $100.7 \pm 0.5$ & $<0.001$ \\
Actual PEF, \% personal best & $75.2 \pm 0.2$ & $75.5 \pm 0.2$ & 0.388 \\
Adherence to ICS, \% & $48.8 \pm 4.5$ & $27.5 \pm 4.9$ & 0.002 \\
Number of quick-relief puffs (past & $41.9 \pm 4.6$ & $21.8 \pm 5.2$ & 0.005 \\
30 days) & & &
\end{tabular}

Values represent mean $\pm S E$ of the mean.

ICS, inhaled corticosteroids; PEF, peak expiratory flow.
These analyses were repeated by excluding 29 children in the PEF feedback group who were between 13 and 15 years old to

\section{Perception of pulmonary function with}

Children who received PEF feedback were more likely to have predictions in the accurate zone than children in the no feedback group $(F(1,189)=12.72$; see table 3$)$. Children who received PEF feedback were less likely to under-perceive respiratory compromise than children in the no feedback group $(1,189)=65.63)$. Children who did not receive PEF feedback were in the danger zone $42 \%$ of the time versus $15 \%$ for the was more common in the PEF feedback group than the no feedback group $(F(1,189)=18.28)$. Use of the AM2 device was iden- 
statistically significant differences in initial perception zone classifications (eg, accurate zone) based on PEF feedback group $(\beta=0.36, p=0.001)$. Examination of the slope results indicated no effect for ethnicity or child's age on perception zone classification. There were no statistically significant changes over time for perception zone classifications in either group. These findings indicate that between-group differences on perception were evident very early during the course of the 6-week perception trial.

\section{PEF feedback and medication use during the perception trial}

Children who received PEF feedback $(n=46)$ had greater adherence to ICS medications during the perception trial than children in the no feedback group $(n=39)$, with age as a covariate in the model $(\mathrm{F}(1,82)=10.08$; see table 3$)$. Children in the PEF feedback group $(n=92)$ also used more puffs of quick-relief medication versus children in the no feedback group $(n=73)$ after controlling for age $(\mathrm{F}(1,161)=7.86)$. These findings did not change when only including children $7-12$ years old.

\section{Perception of pulmonary function and asthma morbidity at baseline}

The danger zone was associated with greater caregiver report of functional morbidity, asthma-related ED visits and hospitalisations in the PEF feedback group (see table 4). This finding suggests that under-perception of respiratory compromise despite PEF prediction feedback is a risk factor linked to asthma morbidity. ED use was high in this sample, as $74.0 \%$ reported at least one ED visit for asthma and $17.3 \%$ reported at least one hospitalisation. No other perception zones were significantly associated with asthma morbidity in either group.

\section{DISCUSSION}

This study shows that inner-city, ethnic minority children are at risk for a dangerous combination of under-perception of respiratory compromise and very poor adherence to ICS medications when measured without PEF prediction feedback. Children who received the PEF prediction feedback intervention had a lower rate of under-perception and higher rates of perceptual accuracy and ICS adherence. This finding might reflect greater attention to asthma perception and management as a result of PEF prediction feedback. Children's attention skills are the strongest predictor of asthma perceptual accuracy. ${ }^{26}$ The surprising finding of greater over-perception and quick-relief use in the PEF feedback condition might also be attributed to higher levels of attention focused on asthma. Given that children who received feedback only used ICS medications slightly less than half of the time, frequent quick-relief medication use may have been the result of seeing low levels of PEF. This finding suggests that significant barriers to ICS medication use (eg, acute, episodic views of asthma) may persist despite improvements in asthma symptom perception. The low rates of under-perception in the PEF feedback group were similar to rates reported for Latino children using the same protocol. ${ }^{13}$ Therefore, previously reported higher rates of accurate perception measured with PEF prediction feedback ${ }^{13} 19$ might underestimate the problem of under-perception of respiratory compromise in more realistic settings that do not involve prediction feedback.

The current study suggests that PEF prediction feedback may be one useful self-management tool in clinical interventions to improve perceptual accuracy and adherence. The act of predicting PEF prior to the manoeuvre may play a critical role, as it requires children to assess their breathing status before receiving the results. Children may view PEF prediction feedback as a game to see how closely their predictions match their actual PEF. The protocol was not simply an exercise in PEF monitoring. Therefore, a system that combines prediction with a PEF monitor might be required to increase perceptual accuracy and ICS adherence. The growth model analyses indicated that children in the feedback condition performed better than the no feedback group in the accurate and danger zones soon after they took the AM2 devices home. The lack of an incremental learning effect across time is consistent with prior studies ${ }^{6} 13$ and suggests that children may show rapid improvement in perceptual accuracy, which is maintained across time. This finding might reflect a ceiling effect that occurs with PEF prediction feedback without additional components, such as asthma education or weekly feedback meetings to discuss perceptual accuracy. Lack of attention to asthma management was demonstrated in the no feedback condition. The absence of significant correlations between children's under-perception of asthma symptoms and parent report of asthma morbidity in the no feedback group might reflect a dangerous situation in which both child and parent are unaware of asthma symptoms, and not seeking healthcare services.

Limitations of this study include the lack of a randomised controlled trial and the difference in age range between the feedback (7-15 years) and no feedback (7-12 years) conditions. However, the only between-group difference on demographics was the child's age. Restricting the age range to 7-12 years in both conditions did not change any results, and the child's age was a covariate in the models. The study relied on convenience samples and community samples should be used in future studies. It is possible that children who were blinded to PEF might have used less effort. However, objective PEF values were not different between the two conditions. Parent report of asthma morbidity was based on retrospective recall. Prospective, longitudinal studies are needed to assess the relationship between children's asthma symptom perception (with and without PEF feedback) and asthma morbidity.

There is great potential to adapt PEF prediction with feedback using the internet, text messaging ${ }^{27} 28$ and smartphone applications, which would create a stimulating and motivating reinforcer. Motivational interviewing with inner-city adolescents to increase adherence to controller medications ${ }^{29}$ might

Table 4 Partial correlations between perception of pulmonary function and baseline asthma morbidity (adjusted for child age)

\begin{tabular}{|c|c|c|c|c|c|c|}
\hline & \multicolumn{3}{|l|}{ PEF feedback } & \multicolumn{3}{|l|}{ No feedback } \\
\hline & $\begin{array}{l}\text { Accurate zone } \\
(n=104)\end{array}$ & $\begin{array}{l}\text { Danger zone } \\
(n=104)\end{array}$ & $\begin{array}{l}\text { Magnification zone } \\
(\mathrm{n}=104)\end{array}$ & $\begin{array}{l}\text { Accurate zone } \\
(n=88)\end{array}$ & $\begin{array}{l}\text { Danger zone } \\
(n=88)\end{array}$ & $\begin{array}{l}\text { Magnification zone } \\
(\mathrm{n}=88)\end{array}$ \\
\hline Asthma functional morbidity ( $\mathrm{p}$ value) & $0.04(0.696)$ & $0.21(0.037)$ & $-0.19(0.059)$ & $-0.02(0.836)$ & $0.04(0.693)$ & $-0.03(0.780)$ \\
\hline ED visits for asthma ( $p$ value) & $-0.11(0.268)$ & $0.27(0.006)$ & $-0.08(0.399)$ & $0.17(0.106)$ & $-0.10(0.374)$ & $-0.08(0.447)$ \\
\hline Hospitalisations for asthma ( $p$ value) & $-0.16(0.118)$ & $0.34(<0.001)$ & $-0.10(0.336)$ & $-0.04(0.687)$ & $0.11(0.309)$ & $-0.10(0.382)$ \\
\hline
\end{tabular}

ED, emergency department; PEF, peak expiratory flow. 
be a complementary intervention to provide additional feedback and increase the rate of ICS adherence. It will also be critical to incorporate caregivers into interventions designed to increase perception of pulmonary function in children to improve asthma management.

Acknowledgements We would like to acknowledge the cooperation from the New York City Health and Hospitals Corporation, Jacobi Medical Center and North Central Bronx Hospital.

Contributors All authors meet the criteria for authorship set forth by the International Committee for Medical Journal Editors. JMF supervised field activities, conducted the literature review and statistical analyses, and drafted initial versions of the manuscript. HK, LM, ML, DS, DS and KW assisted with quality assurance and control and helped direct the implementation of the study. KSA participated in statistical analyses and data interpretation. All authors provided extensive feedback on the entire manuscript.

Funding This study was funded by the American Lung Association (SB-204740N; PI: J Feldman) and National Institute of Child Health and Human Development (1R03HD053355; PI: J Feldman).

Disclaimer The study sponsors had no involvement in study design, data collection, analysis, interpretation of data or the decision to submit the paper for publication.

Competing interests Dr. Serebrisky has received lecture fees from TEVA Respiratory.

Ethics approval Institutional Review Board at Albert Einstein College of Medicine.

Provenance and peer review Not commissioned; externally peer reviewed.

\section{REFERENCES}

1. Magadle R, Berar-Yanay N, Weiner P. The risk of hospitalization and near-fatal and fatal asthma in relation to the perception of dyspnea. Chest 2002;121:329-33.

2. Motomura C, Odajima $\mathrm{H}$, Tezuka J, et al. Perception of dyspnea during acetylcholine-induced bronchoconstriction in asthmatic children. Ann Allergy Asthma Immunol 2009;102:121-4

3. Bjermer L, Diamant Z. Complementary therapy in asthma: inhaled corticosteroids and what? Curr Opin Pulm Med. 2009;15:46-51.

4. Tattersfield AE, Postma DS, Barnes PJ, et al. Exacerbations of asthma: a descriptive study of 425 severe exacerbations. The FACET International Study Group. Am J Respir Crit Care Med 1999;160:594-9.

5. National Heart Lung and Blood Institute. Expert Panel Report 3: Guidelines for the Diagnosis and Management of Asthma. Bethesda, MA, 2007, Contract 08-4051.

6. Silverman BA, Mayer D, Sabinsky R, et al. Training perception of air flow obstruction in asthmatics. Ann Allergy 1987:59:350-4.

7. Janson SL, McGrath KW, Covington JK, et al. Objective airway monitoring improves asthma control in the cold and flu season: a cluster randomized trial. Chest 2010;138:1148-55.

8. Janssens $\mathbf{T}$, Verleden G, De Peuter $S$, et al. Inaccurate perception of asthma symptoms: a cognitive-affective framework and implications for asthma treatment. Clin Psychol Rev 2009;29:317-27.
9. Bem DJ. Self-perception Theory. Advances in Experimental Social Psychology. New York: Academic Press, 1972

10. Moyers TB, Martin T, Houck JM, et al. From in-session behaviors to drinking outcomes: a causal chain for motivational interviewing. J Consult Clin Psychol 2009;77:1113-24.

11. Garg R, Karpati A, Leighton J, et al. Asthma Facts. 2nd edn. New York: Department of Health and Mental Hygiene, 2003.

12. Lara M, Akinbami L, Flores G, et al. Heterogeneity of childhood asthma among Hispanic children: Puerto Rican children bear a disproportionate burden. Pediatrics 2006;117:43-53

13. Fritz GK, McQuaid EL, Kopel SJ, et al. Ethnic differences in perception of lung function: a factor in pediatric asthma disparities? Am J Respir Crit Care Med 2010;182:12-18.

14. Yoos HL, Kitzman H, McMullen A, et al. Symptom perception in childhood asthma: how accurate are children and their parents? J Asthma 2003;40:27-39.

15. Rohan J, Drotar D, McNally K, et al. Adherence to pediatric asthma treatment in economically disadvantaged African-American children and adolescents: an application of growth curve analysis. J Pediatr Psychol 2010;35:394-404.

16. McQuaid EL, Vasquez J, Canino G, et al. Beliefs and barriers to medication use in parents of Latino children with asthma. Pediatr Pulmonol 2009;44:892-8.

17. Halterman $\mathbf{J}$, Yoos $\mathrm{H}$, Kaczorowski $\mathrm{J}$, et al. Providers underestimate symptom severity among urban children with asthma. Arch Pediatr Adolesc Med 2002;156:141-6.

18. Klein RB, Walders N, McQuaid EL, et al. The Asthma Risk Grid: clinical interpretation of symptom perception. Allergy Asthma Proc 2004;25:1-6.

19. Fritz GK, Adams SK, McQuaid EL, et al. Symptom perception in pediatric asthma: resistive loading and in vivo assessment compared. Chest 2007; 132:884-9

20. Feldman JM, McQuaid EL, Klein RB, et al. Symptom perception and functional morbidity across a 1-year follow-up in pediatric asthma. Pediatr Pulmonol 2007:42:339-47.

21. Jentzsch NS, Camargos PA, Colosimo EA, et al. Monitoring adherence to beclomethasone in asthmatic children and adolescents through four different methods. Allergy 2009;64:1458-62.

22. ATS. Standardization of spirometry: 1994. Am J Respir Crit Care Med 1995;152:1107-36.

23. Rosier MJ, Bishop J, Nolan T, et al. Measurement of functional severity of asthma in children. Am J Respir Crit Care Med 1994;149:1434-41.

24. US Department of Health and Human Services. The 2008 HHS Poverty Guidelines. Washington, DC: Department of Health and Human Services, 2008.

25. McOuaid EL, Kopel SJ, Klein RB, et al. Medication adherence in pediatric asthma: reasoning, responsibility, and behavior. J Pediatr Psychol 2003;28:323-33.

26. Koinis-Mitchell D, McQuaid EL, Seifer R, et al. Symptom perception in children with asthma: cognitive and psychological factors. Health Psychol 2009;28:226-37.

27. Petrie KJ, Perry K, Broadbent E, et al. A text message programme designed to modify patients' illness and treatment beliefs improves self-reported adherence to asthma preventer medication. Br J Health Psychol 2012;17:74-84.

28. van der Meer V, Rikkers-Mutsaerts ER, Sterk PJ, et al. Compliance and reliability of electronic PEF monitoring in adolescents with asthma. Thorax 2006;61:457-8.

29. Riekert KA, Borrelli B, Bilderback A, et al. The development of a motivational interviewing intervention to promote medication adherence among inner-city, African-American adolescents with asthma. Patient Educ Couns 2011;82:117-22. 